\title{
Intranasal Hemangiosarcoma in a Dog
}

\author{
Michio FUJITA ${ }^{1) *}$, Yumi TAKAISHI ${ }^{1}$, Daiji YASUDA ${ }^{1,3)}$, Daisuke HASEGAWA ${ }^{1)}$, Akiko TANIGUCHI ${ }^{4)}$, \\ Kimimasa TAKAHASHI ${ }^{2)}$ and Hiromitsu ORIMA ${ }^{1)}$ \\ ${ }^{1)}$ Departments of Veterinary Radiology and ${ }^{2)}$ Veterinary Pathology Nippon Veterinary and Life Science University, 1-7-1 Kyounanchou, \\ Musashinoshi, Tokyo 180-8602, ${ }^{3)}$ Yasuda Animal Hospital, 4181 Totsukacho,Totsukaku, Yokohamashi,Kanagawa 244-0003 and \\ 4) Yamazaki College of Animal Health Technology, 4-7-2 Minami-osawa, Hachioujishi, Tokyo 192-0364, Japan
}

(Received 21 June 2007/Accepted 17 January 2008)

ABSTRACT. Magnetic resonance (MR) was conducted for an 8-year-old, intact male Spitz with sneezing, serous discharge and epistaxis from the left nasal cavity. MR imaging showed a nasal cavity-occupied mass of iso-intensity on T1WI, high-intensity on T2WI and markedly enhanced on contrast-enhanced T1WI at parts of rostal to medial ocular angle in the left cavity. After Surgery and intraoperative radiation, the mass was diagnosed intranasal hemangiosarcoma by histopathology. Although the dog showed the finding, which suggested recurrence after the treatment ending, about 30 months later, it maintained good conditions without evidence of metastasis. KEY WORDS: canine, intranasal hemangiosarcoma, magnetic resonance imaging (MRI).

J. Vet. Med. Sci. 70(5): 525-528, 2008

It has been reported that intranasal tumors account for about $1 \%$ of all tumors in dogs [7]. The onset is about 10 years old on average and though unproved, breeds with a long nose and dogs living in the urban environment and filtrating contaminated substances with their nose seem to have a higher risk $[2,11]$. Patnaik [8] reported that epithelial tumors such as squamous cell carcinoma and adenocarcinoma accounted for about $60 \%$ of intranasal tumors and non-epithelial tumors such as chondrosarcoma, osteosarcoma, and soft tissue sarcoma about $33.7 \%$ in 285 dogs. In general, it is said that the biological behavior of malignant intranasal tumors is characterized by progressive local invasion with lymphatic metastasis at as low as $10 \%$ at the time of diagnosis, but they metastasize to lymph nodes and lungs in $40-50 \%$ at the time of death. Regional lymph nodes and lungs are the main metastatic sites and metastasis to the bones, kidneys, liver, skin, and brain has also been reported.

According to Turek and Lana [16], however, the biological behavior of hemangiosarcoma, neuroendocrine carcinoma, rhabdomyosarcoma, and leiomyosarcoma is poorly understood because of their rarity.

Of them, canine hemangiosarcoma frequently occurs in the spleen and subcutaneous tissue and tends to rapidly metastasize through the bloodstream, and it presents a markedly poor prognosis even if intensive treatment is provided. Here we encountered a dog case that sometimes presented with upper respiratory symptoms such as sneezing and bleeding and serous nasal discharge from the left nasal cavity. MRI findings suggested an intranasal tumor, and surgery and intraoperative radiation were carried out. Later, intranasal hemangiosarcoma was diagnosed by pathology. Since the case was followed-up for about 30 months, we here report the profile and MRI findings of intranasal

\footnotetext{
* Correspondence to: Fujita, M., Department of Veterinary Radiology, Nippon Veterinary and Life Science University, 1-7-1 Kyounanchou, Musashinoshi, Tokyo 180-8602, Japan.

e-mail:mfujita@nvlu.ac.jp
}

hemangiosarcoma.

An intact male Spitz dog of 8 years and 4 months old with a weight of $5.75 \mathrm{~kg}$ admitted to a private animal hospital complaining of paroxysmal sneezing, serous discharge from the left nasal cavity, and snoring sound with an onset of approximately 8 months ago. Because medical treatment with antibiotics and anti-inflammation drugs was not effective and the dog began to present with bleeding from the left nasal cavity associated with an increased frequency of sneezing, the dog was transferred to the Nippon Veterinary and Life Science University Animal Medical Center for further examinations including MRI. On physical examination at referral, the dog had normal activity and appetite but presented with a marked snoring sound as well as serous nasal discharge, although there was no abnormality in the dog's appearance and no palate descensus. A complete blood count showed reproductive anemia [erythrocytopenia (416 $\times 10^{6} / \mu l$; reference range, $\left.550-850 \times 10^{6} / \mu l\right)$, reduced hematocrit (30\%; reference range, 37-55\%), and normal hemoglobin (12.9 g/d $l$; reference range, $12-18 \mathrm{~g} / \mathrm{d} l)]$, leukocytosis $(16,200 / \mu l$, reference range, $6,000-12,000 / \mu l)$, neutrophilia $(8,814 / \mu l$, reference range, $3,000-7,000 / \mu l)$, monocytosis $(1,429 / \mu l$, reference range, $<850 / \mu l)$, and eosionophilia $(1,270 / \mu l$, reference range, $<800 / \mu l)$. Blood chemistry showed no abnormal findings.

A ventrodorsal view of skull X-ray demonstrated decreased permeability of the left nasal cavity and a shift of the nasal septum to the right side at the ipsilateral rostrad area. Thoracic X-ray revealed no evidence of pulmonary metastasis. Of note, blood began to blow up from the left intranasal cavity when the dog acted violently during the Xray examination. In addition, fine needle aspiration cytology was done from the left submandibular lymph nodes, which were found to be slightly swollen and revealed reactive hyperplasia. Afterwards, nasal MRI examination was performed under general anesthesia and controlled ventilation with a respirator. The MRI device used was VISART ${ }^{\mathrm{TM}}$ 

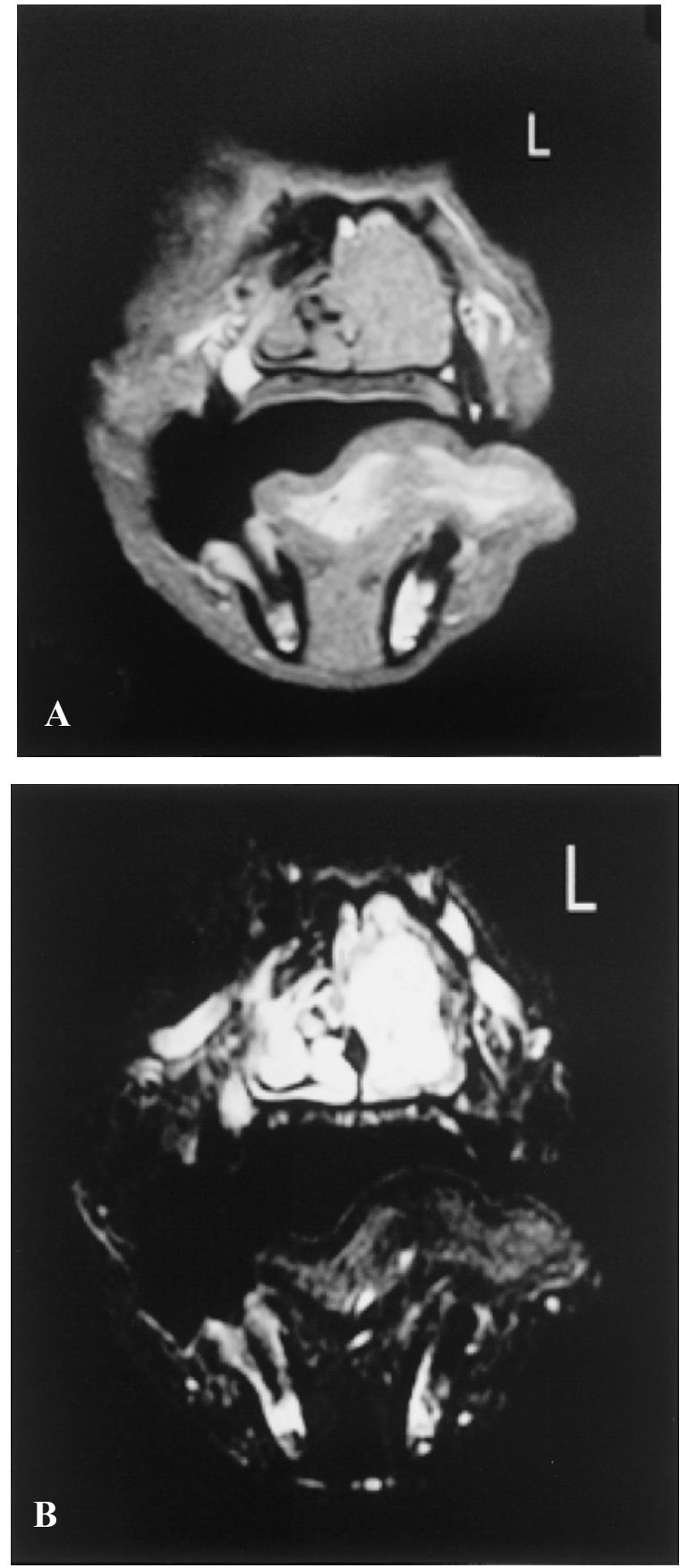

of 1.5 Tesla made by Toshiba Medical Systems Corporation. With this device, we performed T1-weighted imaging (SE method) and T2-weighted imaging (FSE method). The pulse sequence for each imaging was set as TR/TE $=410 / 15$, $\mathrm{TR} / \mathrm{TE}=4000 / 100$, and a slice thickness of $3 \mathrm{~mm}$. MRI revealed a nasal cavity-occupied mass of mainly iso-intensity on T1-weighted imaging (Fig. 1-A) and high-intensity on T2-weighted imaging (Fig. 1-B) at parts of the rostrad to the medial ocular angle in the left nasal cavity. The mass

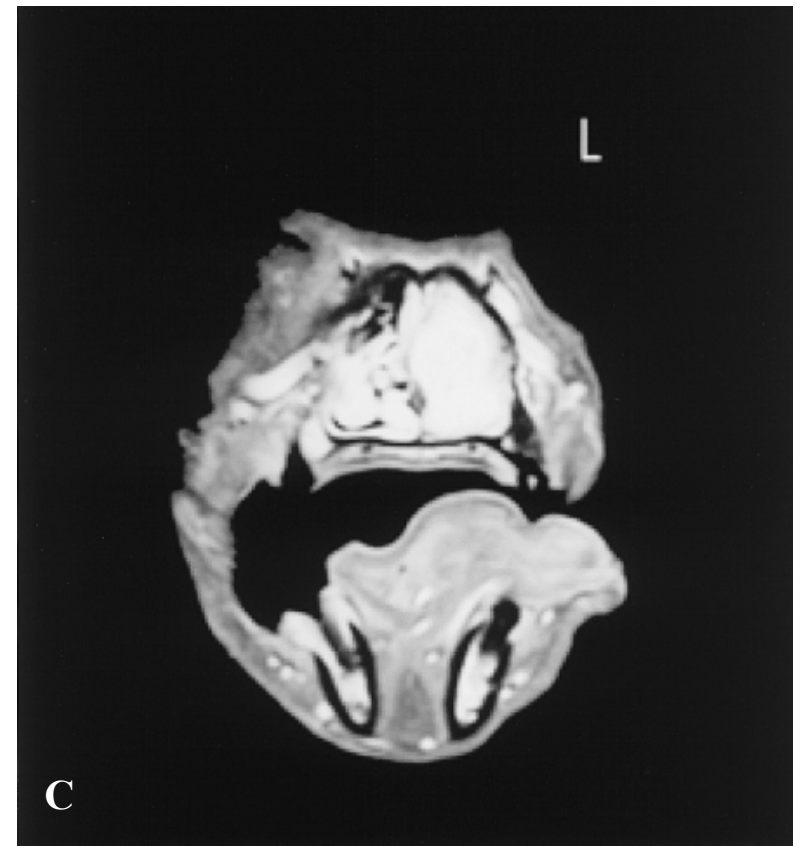

Fig. 1. Transverse T1WI (A), T2WI (B) and contrast-enhanced T1WI (C) of the nasal cavity. On MR imaging, the left nasal nasal cavity area in rostal to medial ocular angle showed a nasal cavity-occupied mass of iso-intensity on T1WI (A), high-intensity on T2WI (B) and markedly enhanced on contrast-enhanced T1WI.

was markedly enhanced on contrast-enhanced T1-weighted transection imaging using $0.2 \mathrm{ml} / \mathrm{kg}$ of Gadolinium DTPA (Omniscan $^{\circledR}$, Daiichi Pharmaceutical Co., Ltd.) (Fig. 1-C). MRI also demonstrated a disappearance of the structure of nasal turbinate at $4-5 \mathrm{~cm}$ caudal part from the rostrad. In addition, a shift of the nasal septum to right at the dorsal rostrad and an invasion to a part of the nasal bone (but not to the right nasal cavity, the frontal sinus, and its surrounding tissues) were found. Since MRI revealed a lesion of iso-inten- 
sity on T1-weighted imaging and high-intensity on T2weighted imaging, it was suggested that the structure was solid and filled with a high water content. In addition, since the tumor showed marked enhancement on contrastenhanced T1-weighted imaging, a shift of the nasal septum to right, and an invasion to a part of the nasal bone, the lesion was highly suspected of a malignant tumor. Therefore, we tried to perform biopsy from the left nasal cavity by inserting a straw-like $16 \mathrm{G}$-caliber pipe connected to a syringe, but were able to obtain only bloody fluid containing a clot probably because of the small caliber. After obtaining an informed consent from the dog's owner, we performed rhinotomy, curetted the content of the left nasal cavity, and irradiated to the exposed intranasal cavity with 20 Gy using orthovoltage X-ray (Radioflex 200EGS-3, $200 \mathrm{kvp}, 5 \mathrm{~mA}$, half value layer $3.42 \mathrm{~mm}$ copper sheet) (made by Rigaku company). After that, we sutured the wound in a usual manner. Some paraffin sections of the resected specimen were stained with hematoxylin and eosin as ordinary, and evaluated histopathologically. There was an anastomosing meshwork of blood-filled channels of varying size. The vascular space was lined by a lot of short-spindle-shaped tumor cells with marked nuclear atypia (Fig. 2-A). With silver reticulin stain, the tumor cells were shown to lie inside the reticulin sheaths blackened by silver. Moreover, immunohistochemical staining for factor VIII revealed positive labeling in some of the tumor cells, leading to a diagnosis of hemangiosarcoma (Fig. 2-B).

Based on the histopathological findings, we recommended adjuvant radiotherapy or chemotherapy to the dog's owner, but could not obtain the owner's agreement. Therefore, the dog was scheduled to be followed-up at the referring animal hospital. Afterwards, sometimes presenting with nasal bleeding, serous nasal discharge, and sneezing, the dog could be managed by symptomatic treatment with antibiotics, anti-inflammation drugs, and hemostatics. Although the dog showed normal activity and appetite for about 28 months after the treatment, swelling at the nasal crest suggested a relapse of the tumor.

Then, the dog was taken to the medical center again about 30 months after the treatment. The affected site was mildly swollen and a reddish soft tissue fragment was confirmed macroscopically in the left nasal cavity. Body weight was $5.95 \mathrm{~kg}$ and not remarkably different from that at the first visit. General conditions were good and normal activity and good appetite was confirmed. We recommended to the dog's owner tissue sampling and MRI for the nasal cavity under sedated conditions, but the owner did not wish for them. Fine needle aspiration biopsy revealed reactive hyperplasia in the left mandibular lymph nodes and a chest $\mathrm{X}$-ray revealed no evidence of metastasis.

Intranasal hemangiosarcoma is a markedly rare tumor, and Patnaik [9] reported that it was found in only seven $(2.5 \%)$ of 285 cases that were diagnosed by autopsy (120 cases) and surgical biopsy (185 cases). Of the seven cases with intranasal hemangiosarcoma, both two autopsied cases showed distant metastasis, but for the others, he did not
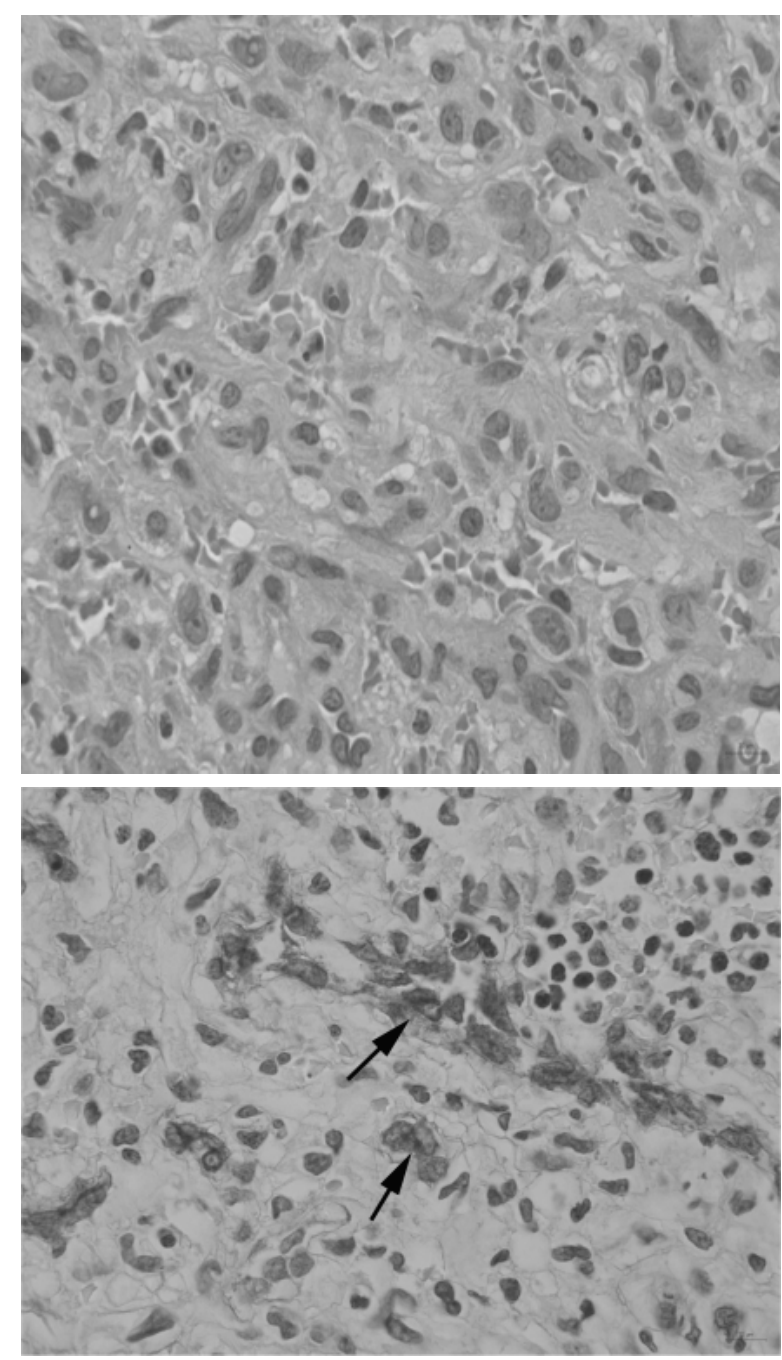

Fig. 2. Histopathology specimens of the intranasal mass of the dog in Fig. 1 (A) The vascular space was lined by a lot of shortspindle-shaped tumor cells with marked nuclear atypia (H.E., bar $=10 \mu \mathrm{m}$ ). (B) Immunohistochemistry staining of the tumor for factor VIII revealed positive labeling in some of the tumor cells (arrow) (bar $=10 \mu \mathrm{m})$.

described concerning the presence of metastasis at the time of diagnosis. Turek and Lana described that its biological behavior was unclear because of the limited number of cases. Hemangiosarcoma occurring at other sites showed a markedly poor prognosis except the cases localized at the dermis, and the median survival time ranged from 19-86 days by surgery alone [1,10] and 141-179 days even by combination with chemotherapy (vincristine, doxorubicin and cyclophosphamide (VAC) or doxorubicin and cyclophosphamide (AC)) after splenectomy $[5,12]$. A one-year survival rate of treated dogs was said to be less than $10 \%$ [17].

In general, the cause of death is metastatic disease. Therefore, it has been reported that radiation therapy may be expected to provide a palliation but would not influence the 
overall survival. Thamm [14] mentioned that radiation therapy was promising as adjuvant therapy for curative treatment after incomplete resection of solitary skin hemangiosarcoma, which has not fully been investigated. In the present case, despite incomplete resection due to intranasal occurrence, only local recurrence was suspected about 30 months after the treatment and there were no findings of distant metastasis. Given this, the metastatic rate of intranasal hemangiosarcoma may be lower than that of hemangiosarcoma occurring in other sites. If so, treatment options effective for other intranasal tumors, such as surgery and postoperative radiation therapy using orthovoltage X-ray or radiation therapy using megavoltage $\mathrm{X}$-ray alone, may significantly prolong the survival time. Further data accumulation is required to clarify these points.

Next, iso-intensity on T1-weighted imaging, high intensity on T2-weighted imaging, and markedly strong intensity on contrast-enhanced T1-weighted imaging were observed as MRI findings of intranasal hemangiosarcoma in this study. Clifford et al. [3] reported that MRI findings of canine hemangiosarcoma in the liver were lower intensity on T1-weighted imaging, higher intensity on T2-weighted imaging, and higher intensity on Gd-enhanced T1-weighted imaging compared with those of normal liver parenchyma. Kippenes et al. [6] mentioned that MRI findings of canine intramedullary hemangiosarcoma were iso-intensity on T1weighted imaging, high intensity on T2-weighted imaging, and heterogeneous but mildly high intensity on Gdenhanced T1-wieghted imaging, and high intensity areas observed on contrast T1-weighted imaging might represent intratumoral fluid when the results on T2-weighted imaging were taken into account.

Sugita et al. [13] reported that MRI findings of human hemangiosarcoma were mostly iso-intensity on T1weighted imaging, marked high intensity on T2-weighted imaging, and low intensity spots by T1- and T2-weighted imaging suggestive of hemorrhagic lesions. Glazebrook et al. [4] showed that MRI findings of hemangiosarcoma in the human mammary gland were markedly high intensity on T2-weighted imaging and this represented the presence of blood vessels abundant in blood flow in the whole tumor. Yang et al. [18] reported that MRI findings in 24 cases of hemangiosarcoma in the human mammary gland were heterogeneously low intensity on T1-weighted imaging, heterogeneously high intensity on T2-weighted imaging, and heterogeneous enhancement on contrast-enhanced T1weighted imaging, which suggested rich blood vessel distribution and hemorrhagic lesions in the tumor.

On the other hand, as MRI findings of human hemangioma, markedly high intensity on T2-weighted imaging and stronger contrast enhancement on contrast T1-weighted imaging were characteristic for this disease [15]. Moreover, it has been said that most of hemangiomas show iso-intensity on T1-wieghted imaging. Image findings of the present case were similar to those of human hemangioma and those reported by Clifford et al. and Kippenes et al., and it was considered that the whole tumor was consisted of the areas with abundant blood flow, and the findings were suggestive of hemangioma or hemangiosarcoma.

In conclusion, we here reported MRI findings and biological behavior of markedly rare intranasal hemangiosarcoma. We are planning to investigate the biological behavior in a larger number of cases and follow-up MRI findings.

\section{REFERENCES}

1. Brown, N.O., Patnaik, A.K. and MacEwen, E.G. 1985. J. Am. Anim. Hosp. 186: 56-58.

2. Bukowski, J.A. and Waterenberg, D. 1998. J. Toxicol. Environ. Health 54: 579-591.

3. Clifford, C.A., Pretorius, E.S., Weisse, C., Sorenmo, K.U., Drobatz, K.J., Siegelman, E.S. and Solmon, J.A. 2004. J. Vet. Intern. Med. 18: 330-338.

4. Glazebrook, K.N., Morton, M.J. and Reynolds, C. 2005. Am. J. Roentgenol. 184: 331-338.

5. Hammer, A.S., Couto, C.G., Filppi, J., Getzy, D. and Shark, K. 1991. J. Vet. Intern. Med. 5: 160-166.

6. Kippenes, H., Gavin, P.R., Bagley, R.S., Silver, G.M., Tucker, R.L. and Sande, R.D. 1999. Vet. Radiol. Ultrasound. 40: 627633.

7. MacEwen, E.G., Withrow, S.J. and Patnaik, A.K. 1977. J. Am. Vet. Med. Assoc. 170: 45-48.

8. Patnail, A.K. 1989. J. Am. Anim. Hosp. 25: 103-114.

9. Patnaik, A.K. 1989. J. Am. Anim. Hosp. 25: 491-497.

10. Pymak, C., McKee, L.J., Goldschmidt, M.H. and Glickman, L.T. 1988. J. Am. Vet. Med. Assoc. 193: 706-712.

11. Reif, J.S. and Cohen, D. 1971. Arch. Environ. Health. 22: 136140.

12. Sorenmo, K.U., Jeglum, K.A. and Helfand, S.C. 1993. J. Vet. Intern. Med. 7: 370-379.

13. Sugita, R., Takezawa, M. and Itinohasama, R. 2002. Radiat. Med. 20: 101-103.

14. Thamm, D.H. 2007. pp. 785-795. In: Small Animal Clinical Oncology, 4th ed. (Withroe, S.J. and Vali, D.M. eds.), Saunders, Missouri.

15. Tonami,H. 1999. pp. 120-121. In: ABC of MRI (Katayama,H. ed.), Nihonishikai, Tokyo (in Japanese).

16. Turek, M.M. and Lana, S.E. 2007. pp. 525-539. In: Small Animal Clinical Oncology, 4th ed. (Withroe, S.J. and Vali, D.M. eds.), Saunders, Missouri.

17. Wood, C.A., Moore, A.S., Gilatto, J.M., Ablin, L.A., Berg, R.J. and Rand, W.M. 1998. J. Am. Anim. Hosp. Assoc. 34: 417-421.

18. Yang, W.T., Hennessy, B.T.J., Dryden, M.J., Valero, V., Hunt, K.K. and Krishnamurthy, S. 2007. Radiology 242: 725-734. 